
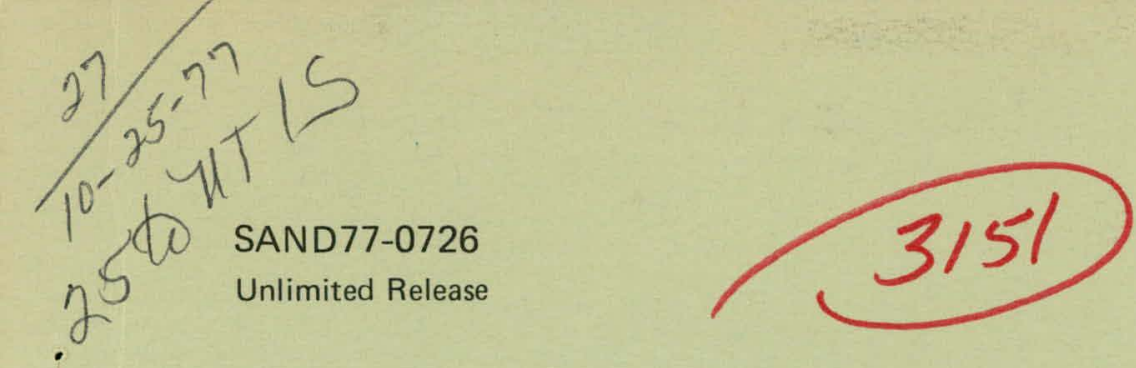

\title{
Accuracy Limitation on Strapdown Calculations in a Coning Environment Due to a Given Approximation to the Body Rate Vector
}

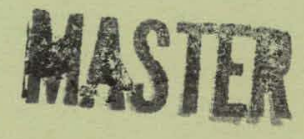

Jerry N. Shinkle

Prepared by Sandia Laboratories, Albuquerque. New Mexico 87115

and Livermore. California 94550 for the United States Energy Research

and Development Administration under Contract AT $(29-1) .789$

Printed September 1977 


\section{DISCLAIMER}

This report was prepared as an account of work sponsored by an agency of the United States Government. Neither the United States Government nor any agency Thereof, nor any of their employees, makes any warranty, express or implied, or assumes any legal liability or responsibility for the accuracy, completeness, or usefulness of any information, apparatus, product, or process disclosed, or represents that its use would not infringe privately owned rights. Reference herein to any specific commercial product, process, or service by trade name, trademark, manufacturer, or otherwise does not necessarily constitute or imply its endorsement, recommendation, or favoring by the United States Government or any agency thereof. The views and opinions of authors expressed herein do not necessarily state or reflect those of the United States Government or any agency thereof. 


\section{DISCLAIMER}

Portions of this document may be illegible in electronic image products. Images are produced from the best available original document. 
Issued by Sandia Laboratories, operated for the United States Energy Research \& Development Administration by Sandia Corporation.

\section{NOTICE}

This report was prepared as an account of work sponsored by the United States Government. Neither the United States nor the United States Energy Research \& Development Administration, nor any of their employees, nor any of their contractors, subcontractors, or their employees, makes any warranty, express or implied, or assumes any legal liability or responsibility for the accuracy, completeness or usefulness of any information, apparatus, product or process disclosed, or represents that its use would not infringe privately owned rights.

Printed in the United States of America

Available from

National Technical Information Service

U. S. Department of Commerce

5285 Port Royal Road

Springfield, VA 22161

Price: Printed Copy $\$ 4.00$; Microfiche $\$ 3.00$ 
SAND77-0726

Unlimited Release

Printed September 1977

\section{ACCURACY LIMITATION ON STRAPDOWN CALCULATIONS \\ IN A CONING ENVIRONMENT DUE TO A GIVEN \\ APPROXIMATION TO THE BODY RATE VECTOR}

Jerry N. Shinkle

Guidance and Control Division 2326

Sandia Laboratories, Albuquerque, NM 87115

\section{ABSTRACT}

The direction cosine matrix-differential equation $[\dot{A}]=[A][\omega]$ can be solved accurately only if a precise representation of the body rate vector $\omega$ is input to the navigation computer. This paper presents an analytical solution for the limits of accuracy that can be ublulied when the $\omega$ vootor is extrarter from polynominal fits to integrating rate sensor output data. The results are applicable to all computational algorithms.

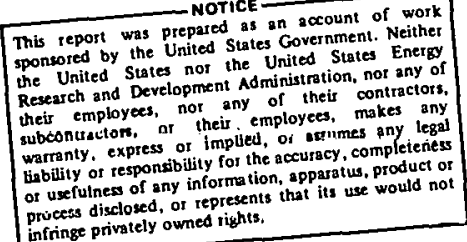

infinge privately owned tights. 


\section{CONTENTS}

\begin{tabular}{lr}
\multicolumn{1}{c}{ CONTENTS } & Page \\
Summary & 7 \\
Introduction & 7 \\
Error Equation Derivation & 10 \\
Drift in Terms of Orientation Vector Error & 13 \\
Truth Solution for Coning Motion & 16 \\
General Solution for Drift & 16 \\
Conclusion & 24 \\
APPENDIX -- Limitations of Some Specific Computational Algorithms & 25
\end{tabular}




\section{ACCURACY LIMITATIONS ON STRAPDOWN CALCULATIONS \\ IN A CONING ENVIRONMENT DUE TO A GIVEN \\ APPROXIMATION TO TIIE BODY RATE VECTOR}

\section{Summary}

A prime requirement of accurate strapdown navigation systems is the accurate computation of the direction cosines $A_{i j}$ which relate the body fixed accelerometer axes to the navigation frame. 'l'he cosines are calculated from some equivalent to the well known matrix differential equation

$$
A=\dot{A}\left[\begin{array}{ccc}
0 & -\omega_{z} & \omega_{y} \\
\omega_{z} & 0 & -\omega_{x} \\
-\omega_{y} & \omega_{x} & 0
\end{array}\right]
$$

where the $\omega_{i}$ are the body angular rates in body coordinates.

In general, however, the $\omega_{i}$ are not truly available and they must be inferred from discrete values of their time integrals (the gyro output data). The accuracy to which this inference can be drawn constitutes a fundamental limitation on the accuracy of the entire system. This paper investigates the extent of this limitation when the $\omega_{i}$ are taken to be the time derivatives of polynomial fits to the gyro output data.

The results are given in Figure 1. They show the limitations on accuracy that are imposed by various degrees of polynomial fit to the gyro data. They represent the standard of excellence for the rest of the computation algorithm.

\section{Introduction}

Accurate integration of the direction cosine differential equations (or the quaternion differential equations) which define the transformation from a body fixed coordinate system to an inertial coordinate system requires an accurate knowledge of the body angular rate vector. Any error in one's knowledge of this vector will lead to a residual attitude drift rate which cannot be overcome by computational procedures, no matter how sophisticated. Such errors usually exist because inertial quality rate sensors are generally of the integrating variety; i. e., their outputs represent the time integral of rate, and one must infer rate from changes in the integral. 


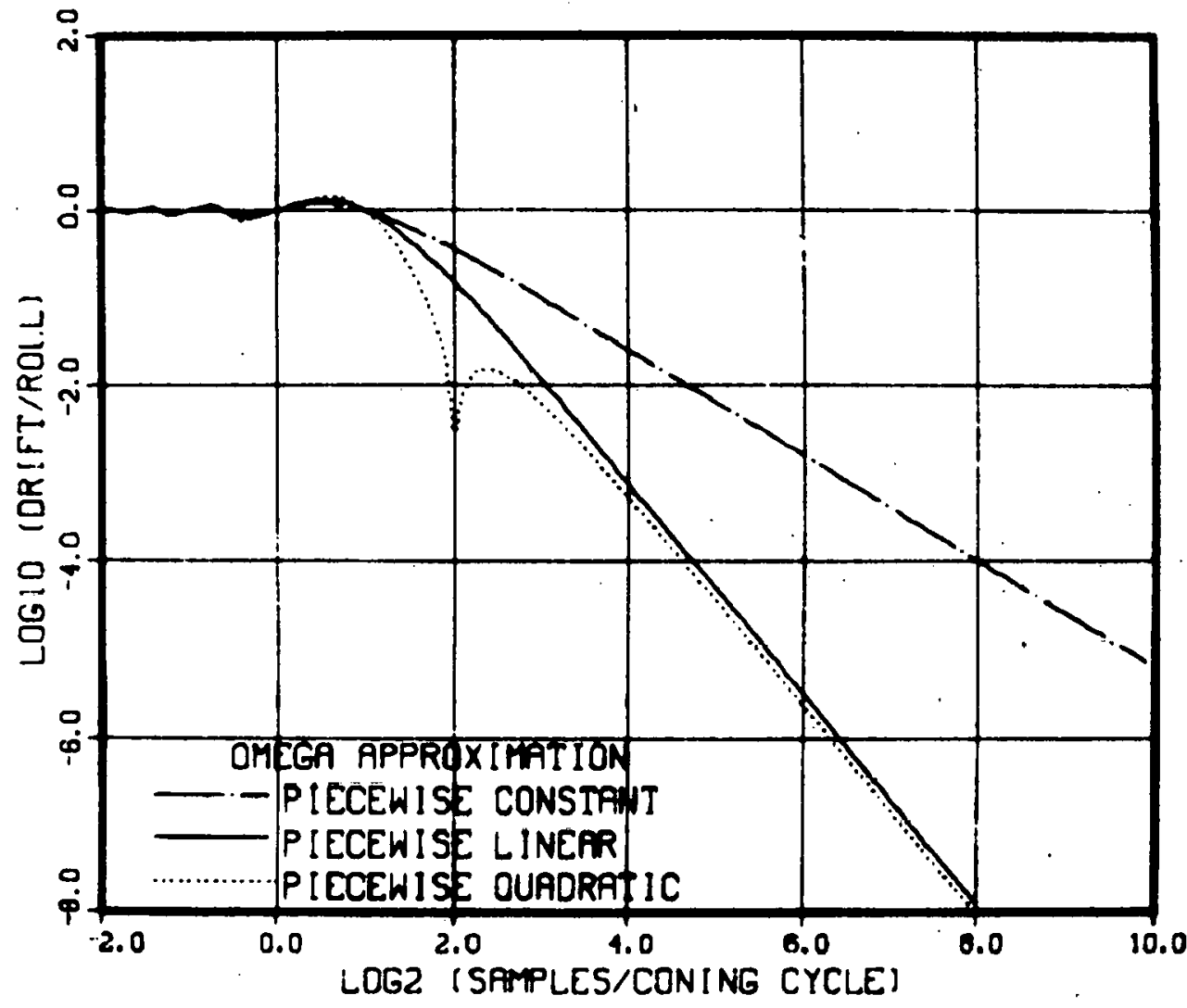

Figure 1. Strapdown Accuracy Limits 
This rate inference can be drawn to various degrees of accuracy. For instance, it can be assumed that the rate is constant over a computational interval,

$$
\underline{\omega}_{\mathrm{o}}=\frac{\Delta \theta}{\Delta t}
$$

or that it is piecewise linear,

$$
\underline{\omega}_{1}=\underline{\omega}(0)+\underline{\dot{\omega}}(0) \Delta t
$$

piecewise quadratic,

$$
\underline{\omega}_{2}=\underline{\omega}(0)+\underline{\dot{\omega}}(0) \Delta t+\frac{1}{2} \underline{\ddot{\omega}}(0) \Delta t^{2}
$$

or, in general,

$$
\underline{\omega}_{m}=\sum_{i=0}^{m} \frac{\omega^{(i)}(0)}{i !} \Delta t^{i} .
$$

The coefficients in these successively improving approximations can be determined by sampling the sensors $1,2,3$, or $m+1$ times, respectively, during a calculation interval and then fitting a curve of the proper degree to the integral data.

The purpose of this paper is to determine the limitations on attitude accuracy that are imposed by these approximations to the rate vector.

Presumably, this task could be accomplished by integrating the usual direction cosine equation

$$
[\dot{A}]=[A][\omega] \text {, }
$$

directly using both the true value of $\underline{\omega}$ and the approximate value $\underline{\hat{\omega}}$. The drift rate could then be determined by comparison. Instead, because of its relative simplicity, we choose here to integrate the Bortz ${ }^{(1)}$ equation for the vehicle orientation vector

$$
\dot{\phi}=\underline{\omega}+\frac{1}{2} \underline{\Phi} \times \underline{\omega}+\frac{1}{12} \Phi \times(\Phi \times \underline{\omega})
$$

and then to determine the drift from the error in $\underline{\phi}$.

(1) J. E. Bortz, "A New Mathematical Formulation for Strapdown Inertial Navigation," IEEE Transactions on Aerospace and Electronic Systems, Vol. AES-7, No. 1, Jan. 1971. 
By definition, the vector $\Phi$ describes a direction and a magnitude such that the body fixed coordinate frame can be made to coincide with the reference frame by means of a rotation of magnitude $\phi$ about the axis $\phi$. The existence of such a vector is guaranteed by a theorem due to Euler. Furthermore, there is a one-to-one correspondence between the components of $\Phi$ and the elements of $[A]$, so $A_{i j}$ can be calculated if $\Phi$ is known.

The procedure will be to derive an error equation by solving Eq. (1) using both the true value of $\underline{\omega}$ and an approximate value $\underline{\hat{\omega}}$. The error in $\Phi$ will then be given by the difference.

\section{Error Equation Derivation}

Take the erroneous value of $\underline{\omega}$ to be given by $\underline{\hat{\omega}}$

$$
\underline{\omega}=\underline{\omega}+\underline{\delta \omega}
$$

and the corresponding erroneous value of the oricntation vector

$$
\Phi=\phi+\delta \phi
$$

where $\delta$ quantities represent the error;

then

$$
\phi-\hat{\Phi}-\underline{\delta \phi}
$$

and

$$
\dot{\psi}-\dot{\dot{\psi}}-\dot{\phi} \phi
$$

Putting Eqs. (2), (4), and (5) into Eq. (1),

$$
\begin{aligned}
\dot{\dot{\phi}}-\underline{\dot{\phi} \phi}= & \hat{\omega}-\underline{\delta \omega}+\frac{1}{2} \dot{\Phi} \times \underline{\hat{\omega}}-\frac{1}{2} \dot{\phi} \times \underline{\delta \omega} \\
& -\frac{1}{2} \delta \underline{\delta \Phi} \times \underline{\omega}+\frac{1}{12} \hat{\Phi} \times(\hat{\Phi} \times \hat{\omega}) \\
& -\frac{1}{12} \hat{\phi} \times(\dot{\Phi} \times \underline{\delta \omega})-\frac{1}{12} \underline{\phi \Phi} \times(\Phi \times \underline{\omega}) \\
& -\frac{1}{12} \hat{\Phi} \times(\underline{\phi} \times \underline{\omega}) ;
\end{aligned}
$$


but

$$
\dot{\dot{\phi}}=\underline{\hat{\omega}}+\frac{1}{2} \hat{\phi} \times \underline{\hat{\omega}}+\frac{1}{12} \hat{\phi} \times(\hat{\phi} \times \underline{\hat{\omega}}) \text {. }
$$

Furthermore, we are interested in the solution only for very small values of $\delta \phi$, so the triple cross products in $\delta \phi$ can be dropped (and $\hat{\phi} \approx \phi$ ). Then, Eq. (6) reduces to

$$
\dot{\delta} \phi=\underline{\sigma \omega}+\frac{1}{2} \phi \times \underline{\delta \omega}+\frac{1}{2} \underline{\delta \phi} \times \underline{\hat{\omega}}+\frac{1}{12} \Phi \times(\phi \times \underline{\delta \omega}) .
$$

The solution to Eq. (8) is complicated by the $\underline{\delta \phi} \times \underline{\omega}$ term on the right hand side. We can perturb a solution by noting that for small $\delta \phi$ and reasonably small $\phi$, to first approximation

$$
\dot{\delta \phi} \approx \underline{\delta \omega}
$$

or

$$
\underline{\delta \phi \approx \delta \theta}=\int \underline{\delta \omega} \mathrm{dt}
$$

then

$$
\dot{\delta} \phi=\underline{\delta \omega}+\frac{1}{2} \Phi \times \underline{\delta \omega}+\frac{1}{2} \underline{\delta \theta} \times \underline{\hat{\omega}}+\frac{1}{12} \phi \times(\underline{\phi} \times \underline{\delta \omega}) .
$$

Consider now the individual terms of Eq. (11) for the classic coning case, for which (Ref. 1)

$$
\Phi=\alpha\left[\begin{array}{c}
\sin \omega t \\
\cos \omega t \\
0
\end{array}\right]
$$

and

$$
\underline{\omega}=\sin \alpha\left[\begin{array}{ccc}
\omega & \cos \omega t \\
-\omega & \sin \omega t \\
\omega & \tan \frac{\alpha}{2}
\end{array}\right]
$$

where $\alpha$ is the coning half angle. 
We define

$$
\underline{\delta \omega}=\sin \alpha\left[\begin{array}{c}
U_{1}(t) \\
U_{2}(t) \\
0
\end{array}\right]
$$

where $U_{1}$ and $U_{2}$ depend on the polynomial approximation to $\underline{\omega}$;

then

$$
\underline{\dot{\omega}}=\underline{\omega}+\underline{\delta \omega} .
$$

and

$$
\underline{\delta \theta}=\sin \alpha\left[\begin{array}{c}
\int \mathrm{U}_{1} \mathrm{dt} \\
\int \mathrm{U}_{2} \mathrm{dt} \\
.0
\end{array}\right] \equiv \sin \alpha\left[\begin{array}{c}
\mathrm{V}_{1}(\mathrm{t}) \\
\mathrm{V}_{2}(\mathrm{t}) \\
0 .
\end{array}\right] .
$$

Note that $\underline{\delta \theta}$ vanishes at the end of each gyro sampling interval (we are assuming perfect gyros).

Using the above, we obtain

$$
\Phi \times \underline{\delta \omega}=\alpha \sin \alpha\left[\begin{array}{c}
0 \\
0 \\
U_{2} \sin \omega t-U_{1} \cos \omega t
\end{array}\right]
$$

and

$$
\underline{\delta \theta} \times \underline{\hat{\omega}}=\sin ^{2} \alpha\left[\begin{array}{c}
\omega \tan \frac{\alpha}{2} v_{2}(t) \\
-\omega \tan \frac{\alpha}{2} v_{1}(t) \\
V_{1}\left(U_{3}-\omega \sin \omega t\right)-V_{2}\left(U_{1}+\omega \cos \omega t\right)
\end{array}\right] \text {. }
$$

We see then that the terms of Eq. (11) for $\Phi \dot{\phi}$ are of the order $\alpha, \alpha^{2}$, and $\alpha^{3}$. The integrals of the various terms contribute to the error in $\Phi$ in these same orders.

'I'he first term is of order $\alpha$, but it contributes nothing to the average drift because the integral vanishes at the end of each gyro sampling interval. The single cross product terms are of order $\alpha^{3}$ on the numbers 1 and 2 axes and order $\alpha^{2}$ on the 3 axis. The double cross product . terms of order $\alpha^{3}$ and have no 3 axis components (because $\phi \times \underline{\delta}$ has no 1 or 2 axis components). 
In addition to the 1 and 2 axis components being of higher order in the small angle $\alpha$ it is also possible to show that they are cyclic in time with a frequency equal to the coning frequency. Hence, the long term error will grow only on the 3 axis. Equation (11) can be integrated over a cycle to give,

$$
2 \Delta \delta \phi_{3}=\oint[\phi \times \underline{\delta \omega}+\underline{\delta \theta} \times \underline{\hat{\omega}}]_{3} \mathrm{dt} ;
$$

but

$$
\Phi \times \underline{\delta \omega}+\underline{\delta \theta} \times \underline{\hat{\omega}}=\Phi \times(\underline{\hat{\omega}}-\underline{\omega})+\underline{\delta \theta} \times \underline{\hat{\omega}}=(\Phi+\underline{\delta \theta}) \times \underline{\hat{\omega}}-\Phi \times \underline{\omega}
$$

and

$$
\oint \Phi \times \underline{\omega} d t=2 \int(\dot{\phi}-\underline{\omega}) d t=2(\underline{\Delta}-\underline{\Delta \theta})_{c y c l e} .
$$

Now for

$$
\underline{\phi}_{\text {cycle }}=\underline{0} ;
$$

and

$$
\underline{\Delta \theta}_{\text {cycle }}=\left[\begin{array}{c}
0 \\
0 \\
2 \pi(1-\cos \alpha)
\end{array}\right] \text {, }
$$

we have, for Eq. (19),

$$
2 \Delta \delta \phi_{3}=4 \pi(1-\cos \alpha)+\oint[(\phi+\delta \theta) \times \underline{\hat{\omega}}]_{3} d t .
$$

Equation (24) is the equation to be solved for the error in $\Phi$ that accumulates over one coning cycle.

Drift in Terms of Orientation Vector Error

Consider the body fixed vector $\underline{r}^{B}$ and the quaternion $Q$ which transforms the vector's components to the inertial frame according to

$$
\underline{r}=Q \underline{r}^{B} \bar{Q}
$$

where

$$
Q=\left[Q_{1}, Q_{2}, Q_{3}, Q_{4}\right]
$$


and $\bar{Q}$ is the conjugate quaternion

$$
\bar{Q}=\left[Q_{1},-Q_{2},-Q_{3},-Q_{4}\right]
$$

The vector $\underline{r}^{B}$ is transformed to the navigation frame by the erroneous value of $Q$

$$
\underline{\mathbf{r}}^{\mathbf{N}}=\underline{\hat{Q}}^{\mathrm{B}} \overline{\overline{\mathbf{Q}}},
$$

so that

$$
\underline{\underline{r}}^{B}=\bar{Q}_{\underline{r}} \mathbf{Q}=\overline{\hat{Q}}_{\underline{\underline{r}}} \underline{\underline{Q}}^{\hat{Q}}
$$

or

$$
\begin{aligned}
\underline{r}^{I} & =Q \overline{\hat{Q}} \underline{\underline{r}} \underline{\mathrm{Q}}^{\mathrm{Q}} \overline{\mathbf{Q}} \\
& \equiv \mathrm{D} \underline{r}^{\mathrm{N}} \overline{\mathrm{D}},
\end{aligned}
$$

where $D$ is the drift quaternion.

$$
\mathrm{D}=\mathrm{Q} \overline{\hat{Q}}
$$

Now any quaternion can be written in terms of the corresponding orientation vector

$$
Q=\left[\cos \frac{\phi}{2}, \frac{\phi_{1}}{\phi} \sin \frac{\phi}{2}, \frac{\phi_{2}}{\phi} \sin \frac{\phi}{2}, \frac{\phi_{3}}{\phi} \sin \frac{\phi}{2}\right] ;
$$

then

$$
\begin{aligned}
& \hat{Q}=\left[\cos \frac{\hat{\phi}}{2}, \frac{\hat{\phi}_{1}}{\hat{\phi}} \sin \frac{\hat{\phi}}{2}, \frac{\hat{\phi}_{2}}{\hat{\phi}} \sin \frac{\hat{\phi}}{2}, \frac{\hat{\phi}_{3}}{\hat{\phi}} \sin \frac{\hat{\phi}}{2}\right] . \\
& \mathrm{D}=\left[\cos \frac{\lambda}{2}, \frac{\lambda_{1}}{\lambda} \sin \frac{\lambda}{2}, \frac{\lambda_{2}}{\lambda} \sin \frac{\lambda}{2}, \frac{\lambda_{3}}{\lambda} \sin \frac{\lambda}{2}\right],
\end{aligned}
$$

where $\lambda$ is the drift orientation vector. Since $\lambda$ is our sole interest here (we do not care what direction the drift takes), we need only calculate $D_{1}$, which is given by 


$$
\begin{aligned}
D_{1} & =[Q \overline{\hat{Q}}]_{1}=Q_{1} \overline{\hat{Q}}_{1}-Q_{2} \overline{\hat{Q}}_{2}-Q_{3} \overline{\hat{Q}}_{3}-Q_{4} \overline{\hat{Q}}_{4} \\
& =\sum_{i} Q_{i} \hat{Q}_{i} \\
& =\cos \frac{\phi}{2} \cos \frac{\hat{\phi}}{2}+\sin \frac{\phi}{2} \sin \frac{\hat{\phi}}{2}\left[\frac{\phi_{1} \hat{\phi}_{1}+\phi_{2} \hat{\phi}_{2}+\phi_{3} \hat{\phi}_{3}}{\phi \hat{\phi}}\right] \\
& =\cos \frac{\phi}{2} \cos \frac{\hat{\phi}}{2}+\sin \frac{\phi}{2} \sin \frac{\hat{\phi}}{2}\left[\frac{\phi \cdot \hat{\phi}}{\phi \hat{\phi}}\right] .
\end{aligned}
$$

The bracketed term in Eq. (38) is just the cosine of the angle between $\hat{\phi}$ and $\Phi$ which, for $\delta \phi \perp \phi$ and $\delta \phi \ll \phi$, is

$$
\frac{\phi \cdot \hat{\phi}}{\phi \hat{\phi}}=1-\frac{1}{2}\left(\frac{\delta \phi}{\phi}\right)^{2}
$$

then

$$
\begin{aligned}
D_{1} & =\cos \frac{\hat{\phi}-\phi}{2}-\frac{1}{2}\left(\frac{\delta \phi}{\phi}\right)^{2} \sin \frac{\phi}{2} \sin \frac{\hat{\phi}}{2} \\
& \approx 1-\frac{1}{2}\left(\frac{\delta \phi}{2}\right)^{2} \\
& \approx \cos \frac{\delta \psi}{2} \\
& =\cos \frac{\lambda}{2} .
\end{aligned}
$$

Hence, the drift angle $\lambda$ is just $\delta \phi$. 


\section{Truth Solution for Coning Motion}

Bortz (Ref. 1 ) shows that the $\Phi$ and $\underline{\omega}$ vectors can be given by

$$
\begin{aligned}
& \underline{\underline{ }}=\left[\begin{array}{ccc}
\alpha & \sin & \omega t \\
\alpha & \cos & \omega t \\
0 &
\end{array}\right] \\
& \underline{\omega}=\omega \sin \alpha\left[\begin{array}{cc}
\cos \omega t \\
-\sin & \omega t \\
\tan & \frac{\alpha}{2}
\end{array}\right]
\end{aligned}
$$

for the case of coning motion of angular frequency $\omega$ and coning half angle $\alpha$.

\section{General Solution for Drift}

We return now to the solution of Eq. (24) for the drift over one cycle.

Let

$$
\begin{aligned}
& m \equiv \text { the degree of the polynomial fit to } \underline{\omega} \\
& h \equiv \text { the gyro sampling interval. }
\end{aligned}
$$

During the $\mathrm{n}^{\text {th }}$ orientation vector update interval $[(m+1) \mathrm{h}$ in duration $]$,

$$
\underline{\dot{\omega}}_{n}=\sum_{i=0}^{m} \eta^{i} \frac{\hat{\hat{\omega}}_{\text {on }}^{(i)}}{i !} \text {, }
$$

where $\eta=t-t_{n}$

$$
t_{n}-l_{1} \text { time at the beginning of the intcrval, }
$$

and the superscript index in parenthesis refers to the ith time derivative. The integral of $\underline{\omega}_{n}$ results in the erroneous value of the gyro vector $\underline{\theta}$.

$$
\hat{\underline{\theta}}_{n}=\sum_{i=0}^{m} \eta^{i+1} \frac{\ddot{\hat{\omega}}_{\text {on }}^{(i)}}{(i+1) !}
$$


and

$$
\underline{\delta \theta}_{n}=\sum_{i=0}^{m} \eta^{i+1} \frac{\underline{\hat{\omega}}_{\text {on }}^{(i)}}{(i+1) !}-\underline{\theta}_{n}
$$

where

$$
\underline{\theta}_{n}=\int_{t_{n}}^{t_{n}+\eta} \underline{\omega}_{n} d t=\sin \alpha\left[\begin{array}{c}
\sin \omega\left(t_{n}+\eta\right)-\sin \omega t_{n} \\
\cos \omega\left(t_{n}+\eta\right)-\cos \omega t_{n} \\
\eta \tan \frac{\alpha}{2}
\end{array}\right] ;
$$

then

$$
\begin{aligned}
& \underline{\phi}+\underline{\theta \theta}_{\mathrm{n}}=\alpha\left[\begin{array}{c}
\sin \omega\left(\mathrm{t}_{\mathrm{n}}+\eta\right) \\
\cos \omega\left(\mathrm{t}_{\mathrm{n}}+\eta\right) \\
0
\end{array}\right]-\sin \alpha\left[\begin{array}{c}
\sin \omega\left(\mathrm{t}_{\mathrm{n}}+\eta\right)-\sin \omega \mathrm{t}_{\mathrm{n}} \\
\cos \omega\left(\mathrm{t}_{\mathrm{n}}+\eta\right)-\cos \omega \mathrm{t}_{\mathrm{n}} \\
\eta \tan \frac{\alpha}{2}
\end{array}\right] \\
&+\sum_{\mathrm{i}=0}^{m} \eta^{\mathrm{i}+1} \frac{\underline{\hat{\omega}}_{\mathrm{on}}^{(\mathrm{i})}}{(\mathrm{i}+1) !}
\end{aligned}
$$

and for $\alpha \approx \sin \alpha$

$$
\Phi+\frac{\delta \theta}{n}=\alpha\left[\begin{array}{cc}
\sin & \omega t_{n} \\
\cos & \omega t_{n} \\
\eta \tan & \frac{\alpha}{2}
\end{array}\right]+\sum_{i=0}^{m} \frac{\eta^{i+1} \underline{\hat{\omega}}_{o n}^{(i)}}{(i+1) !} .
$$

The integrand in Eq. (24) is

$$
\begin{aligned}
{\left[\left(\underline{\phi}+\underline{\delta}_{n}\right) \times \hat{\hat{\omega}}_{\mathrm{n}}\right]_{3}=} & \alpha\left[\hat{\omega}_{\mathrm{n} 2} \sin \omega \mathrm{t}_{\mathrm{n}} \cdots \hat{\omega}_{\mathrm{n} 1} \cos \omega \mathrm{t}_{\mathrm{n}}\right] \\
& +\left[\sum_{\mathrm{i}=0}^{111} \frac{\eta^{i+1} \underline{\hat{\omega}}_{\mathrm{on}}^{(i)}}{(\mathrm{i}+1) !} \times \sum_{j=0}^{\mathrm{m}} \frac{\eta^{\mathrm{j}} \underline{\hat{\omega}}_{\mathrm{on}}^{(j)}}{j !}\right]_{3}
\end{aligned}
$$




$$
\begin{aligned}
= & \alpha \sum_{i=0}^{m}\left[\hat{\omega}_{o n 2}^{(i)} \sin \omega t_{n}-\hat{\omega}_{o n 1}^{(i)} \cos \omega t_{n}\right] \frac{\eta^{i}}{(i) !} \\
& +\sum_{i=0}^{m} \sum_{j=0}^{m}\left[\hat{\omega}_{o n 1}^{(i)} \hat{\omega}_{o n 2}^{(j)}-\hat{\omega}_{o n 2}^{(i)} \hat{\omega}_{o n 1}^{(j)}\right] \frac{\eta^{i+j+1}}{(i+1) ! j !}
\end{aligned}
$$

We now show that the expression given in Eq. (52) is independent of the index $n$; that is, the drift over any one update interval is the same as for any other update interval.

Let the elements in the expansion of the $\underline{\hat{\omega}}$ vector be determined as linear combinations of the gyro sample vector $\underline{\delta}$.

$$
\underline{\hat{\omega}}_{\text {on }}^{(i)}=\sum_{k=1}^{m+1} A_{i k} \underline{\delta}_{n k} \text {, }
$$

where

$$
\underline{\delta}_{n k}=\alpha\left[\begin{array}{c}
\sin \omega\left(t_{n}+k h\right)-\sin \omega t_{n} \\
\cos \omega\left(t_{n}+k h\right)-\cos \omega t_{n} \\
k \eta \tan \frac{\alpha}{2}
\end{array}\right]
$$

is the kth gyro sample in the nth update interval, and the $A_{i l f}$ are to be determined. Then,

$$
\begin{aligned}
\hat{\omega}_{\text {on2 }}^{(i)} \sin \omega t_{n}-\hat{\omega}_{\text {on } 1}^{(i)} \cos \omega t_{n}= & \sum_{k=1}^{m+1} \alpha A_{i k}\left\{\sin \omega t_{n}\left[\cos \omega\left(t_{n}+k h\right)-\cos \omega t_{n}\right]\right. \\
& \left.-\infty \alpha \omega_{n}\left[\sin \omega\left(t_{n}+k h\right)-\sin \omega t_{n}\right]\right\} \\
& \cdot \\
= & -\sum_{k=1}^{m+1} \alpha A_{i k} \sin k \omega h
\end{aligned}
$$

$\therefore$ the first summation in Eq. (52) is independent of $n$. 
Similarly,

$$
\begin{aligned}
\cdot \hat{\omega}_{\mathrm{on} 1}^{(\mathrm{i})} \omega_{\mathrm{on} 2}^{(j)}-\hat{\omega}_{\mathrm{on} 2}^{(\mathrm{i})} \hat{\omega}_{\mathrm{on} 1}^{(j)} & =\sum_{\mathrm{k}=1}^{m+1} \sum_{\ell=1}^{m+1}\left[A_{\mathrm{ik}} \delta_{n k 1} A_{j \ell} \delta_{n \ell 2}-A_{\mathrm{ik}} \delta_{n k 2} A_{j \ell} \delta_{n \ell 1}\right] \\
& =\sum_{\mathrm{k}=1}^{m+1} \sum_{\ell=1}^{m+1} A_{i k} A_{j \ell}\left[\delta_{n k 1} \delta_{n \ell 2}-\delta_{n k 2} \delta_{n \ell 1}\right] ;
\end{aligned}
$$

but,

$$
\begin{aligned}
\delta_{n k 1} \delta_{n \ell 2}-\delta_{n k 2} \delta_{n \ell 1}= & \alpha^{2}\left[\sin \omega\left(t_{n}+k h\right)-\sin \omega t_{n}\right]\left[\cos \omega\left(t_{n}+\ell h\right)-\cos \omega t_{n}\right] \\
& -\alpha^{2}\left[\cos \omega\left(t_{n}+k h\right)-\cos \omega t_{n}\right]\left[\sin \omega\left(t_{n}+\ell h\right)-\sin \omega t_{n}\right] \\
= & \alpha^{2}[\sin (k-\ell) \omega h+\sin \ell \omega h-\sin k \omega h]
\end{aligned}
$$

again, independent of $n$.

We now have

$$
\begin{aligned}
\left(\underline{\phi}+\underline{\delta \theta}_{n}\right) \times \underline{\hat{\omega}}_{n}= & -\alpha^{2} \sum_{i=0}^{m} \prod_{i !}^{i} \sum_{j=1}^{m+1} A_{i j} \sin j \omega h \\
& +\alpha^{2} \sum_{i=0}^{m} \sum_{j=0}^{m} \frac{\eta^{i+j+1}}{(i+1) ! j !} \sum_{k=1}^{m+1} \sum_{\ell=1}^{m+1} A_{i k} A_{j \ell}[\sin (k-\ell) \omega h \\
& +\sin \ell \omega h-\sin k \omega h]
\end{aligned}
$$


also

$$
\begin{aligned}
\int_{0}^{2 \pi / \omega} \cdot[(\phi+\underline{\theta}) \times \underline{\hat{\omega}}] \mathrm{dt}= & \frac{2 \pi}{(m+1) \omega h} \int_{0}^{(m+1) h}[(\phi+\delta \theta) \times \underline{\hat{\omega}}] \mathrm{d} \eta \\
= & \frac{2 \pi \alpha^{2}}{(m+1) \omega h} \sum_{i=0}^{m} \frac{(m+1)^{i+1} h^{i+1}}{(i+1) !} \sum_{j=1}^{m+1} A_{i j} \sin j \omega h \\
& +\frac{2 \pi \alpha^{2}}{(m+1) \omega h} \sum_{i=0}^{m} \sum_{j=0}^{m} \frac{(m+1)^{i+j+2} h^{i+j+2}}{(i+1) ! j !(i+j+2)} \sum_{k=1}^{m} \sum_{l=1}^{m}[\sin (k-\ell) \omega h \\
& +\sin \ell \omega h-\sin k \omega h] .
\end{aligned}
$$

As indicated by Eqs. (13) and (45), the roll axis rate sensor sees a constant rate input even though the vehicle returns to the same orientation once each coning cycle. This is possible, of course, because of the noncommutativity of finite rotations. It is precisely this roll rate which causes computational drift. In fact, if no corrections were to be made for noncommutativity, the computational drift would be equal to the roll angle. Hence, a figure of merit for any computational algorithm might be the ratio of computational drift to actual roll.

Suppose we now change the notation in Eq. (24) to

$$
\Delta \delta \phi_{3} \equiv \mathrm{RRIFT}
$$

and normalize the equation with respect to the actual vehicle roll

$$
\begin{aligned}
\text { ROLL } & =\frac{2 \pi}{\omega} \times \text { ROLL RATE } \\
& =\frac{2 \pi}{\omega} \cdot \omega(1-\cos \alpha)=2 \pi(1-\cos \alpha) \approx \pi \alpha^{2}
\end{aligned}
$$


Using Eqs. (59), (60), and (61) in Eq. (24),

$$
\begin{aligned}
\frac{\text { DRIFT }}{\text { ROLL }}= & 1-\sum_{i=0}^{m} \frac{(m+1)^{i}}{(i+1) !} \sum_{j=1}^{m+1} b_{i j} \frac{\sin j \omega h}{\omega h}+ \\
& \sum_{i=0}^{m} \sum_{j=0}^{m} \frac{(m+1)^{i+j+1}}{(i+1) ! j !(i+j+2)} \sum_{j=1}^{m+1} \sum_{\ell=1}^{m+1} b_{i k} b_{j \ell} \\
& {\left[\frac{\sin (k-\ell) \omega h+\sin \ell \omega h-\sin k \omega h}{\omega h}\right], }
\end{aligned}
$$

where

$$
b_{i j}=A_{i j} h^{i+1}
$$

Because the second double summation in Eq. (62) is antisymmetric with respect to $\mathrm{k}$ and $\ell$, the equation can be written

$$
\begin{aligned}
\frac{\text { DRIFT }}{\text { ROLL }}= & 1-\sum_{i=0}^{m} \frac{(m+1)^{i}}{(i+1) !} \sum_{j=1}^{m+1} b_{i j} \frac{\sin j \omega h}{\omega h}+ \\
& \sum_{i=0}^{m} \sum_{j=0}^{m} \frac{(m+1)^{i+j+1}}{(i+1) ! j !(i+j+2)} \sum_{k=1}^{m} \sum_{\ell=k+1}^{m+1}\left(b_{i k} b_{j \ell}-\right. \\
& \left.b_{i \ell} b_{j k}\right)[\sin \ell \omega h-\sin k \omega h l-\sin (\ell-k) \omega h] .
\end{aligned}
$$

It remains now only to evaluate the $b_{i j}{ }^{*}$ This could be done in general form, but the $b_{i j}$ themselves turn out to be sums and the term in Eq. (63) would become triple and sextuple summations. Elegant perhaps, but too laborious. Let us just evaluate them for specific instances.

Piecewise Constant $\omega(m=0)$

For this simple case, the gyro vector is

$$
\underline{\delta}_{1}=\underline{\hat{\omega}}_{\mathrm{h}}
$$

or 


$$
\underline{\hat{\omega}}=\frac{1}{\mathrm{~h}} \underline{\delta}_{1}
$$

hence,

$$
\begin{aligned}
& A_{01}=1 / h \\
& b_{01}=1 .
\end{aligned}
$$

Equation (63) then gives

$$
\frac{\text { DRIFT }}{\text { ROLL }}=1-\frac{\sin \omega h}{\omega h}
$$

for $m=0$.

Piecewise Linear $\underline{\omega}(\mathrm{m}=1)$

The gyro vectors are

$$
\left.\begin{array}{l}
\underline{\delta}_{1}=\underline{\hat{\omega}}_{0} \mathrm{~h}+\frac{1}{2} \dot{\hat{\hat{\omega}}}_{0} \mathrm{~h}^{2} \\
\underline{\delta}_{2}=2 \underline{\hat{\omega}}_{0} \mathrm{~h}+2 \dot{\hat{\omega}}_{0} \mathrm{~h}^{2}
\end{array}\right\} .
$$

giving

$$
\left.\begin{array}{l}
\underline{\hat{\omega}}_{0}=\left(\underline{\underline{\delta}}_{1}-\frac{1}{2} \underline{\delta}_{2}\right) / \mathrm{h} \\
\underline{\dot{\omega}}_{0}=\left(-2 \underline{\delta}_{1}+\underline{\delta}_{2}\right) / \mathrm{h}^{2}
\end{array}\right\}
$$

and $\mathrm{Eq} .(63)$ evaluates to

$$
\frac{\text { DRIFT }}{\text { ROLL }}=1-\frac{4}{3} \frac{\sin \omega h}{\omega h}+\frac{1}{3} \frac{\sin 2 \omega h}{2 \omega h} \text {. }
$$


$\underline{\text { Piecewise Quadratic } \underline{\omega}(\mathrm{m}=2)}$

Simply stating results:

$$
\begin{aligned}
& b_{i j}=\left[\begin{array}{ccc}
3 & -\frac{3}{2} & \frac{1}{3} \\
-5 & 4 & -1 \\
3 & -3 & 1
\end{array}\right] \quad \begin{array}{r}
i=0,1,2 \\
j=1,2,3
\end{array} \\
& \frac{\text { DRIFT }}{\text { ROLL }}=1-\frac{65}{40} \frac{\sin \omega h}{\omega h}+\frac{32}{40} \frac{\sin 2 \omega h}{2 \omega h}-\frac{7}{40} \frac{\sin 3 \omega h}{3 \omega h}
\end{aligned}
$$

The above results can be expressed in terms of the number of gyro samples per coning cycle (N) by substituting

$$
\frac{2 \pi}{N}=\omega h \quad
$$

The drift results are plotted against $\mathrm{N}$ in Figure 1.

The significant aspects of the plot are as follows.

(1) The drift quickly approaches an asymmetric value as the number of samples per cycle increases. The asymptotes can be obtained easily by expanding the trig functions in the analytical results.

They are

Piecewise Constant

$$
\frac{\text { DRIFT }}{\text { ROLL }} \rightarrow \frac{2 \pi^{2}}{3 N^{2}}
$$

Piecewise Linear

$$
\frac{\text { DRIFT }}{\text { ROLL }}-\frac{8 \pi^{4}}{15 N^{4}}
$$

Piecewise Quadratic

$$
\frac{\text { DRIFT }}{\text { ROLL }} \rightarrow \frac{6 \pi^{4}}{15 N^{4}}
$$


(2) The quadratic approximation gives significant improvement only up to about 8 samples per cycle. Beyond that the improvement is only about $25 \%$.

\section{Conclusion}

A very large improvement in accuracy can be obtained by changing from the constant approximation to the linear approximation. The small additional improvement that is obtained by going to the quadratic approximation is not justified in view of the increased calculational complexity.

For the linear case, and for more than about four samples per cycle, the standard of excellence for computing algorithm comparisons is

$$
\frac{\text { DRIFT }}{\text { ROLL }}=\frac{8 \pi^{2}}{15 N^{4}} \text {. }
$$




\title{
APPENDIX
}

\section{Limitations of Some Specific Computational Algorithms}

\author{
Jordan Algorithm
}

The computational algorithm proposed by Jordan ${ }^{(2)}$ is based on the Bortz equation for the orientation vector. After fitting a quadratic to two consecutive gyro displacement vectors, $\delta_{1}$ and $\delta_{2}$, he shows that the orientation vector can be updated according to

$$
\Phi_{\mathrm{k}+1}=\Phi_{\mathrm{k}}+\underline{\delta}_{2}+\frac{1}{2} \Phi_{\mathrm{k}} \times \underline{\delta}_{2}+\frac{2}{3}\left(\underline{\delta}_{1} \times \underline{\delta}_{2}\right) \text {. }
$$

The orientation vector is updated every two gyro samples until there is a requirement for an updated set of direction cosines. The cosines are then updated using the current value of $\Phi$ according to

$$
A_{k+1}=A_{k} e^{[\phi]_{k}}
$$

Since the algorithm is based on a piecewise quadratic fit to the gyro data, its accuracy is limited by the piecewise linear $\underline{\omega}$ theory of this paper.

\section{Third Order Direction Cosine Expansion}

A Taylor's Series expansion of the direction cosines can be written as

$$
A_{k+1}=A_{k}+\dot{A}_{k} \eta+\frac{1}{2} \ddot{A}_{k} \eta^{2}+\frac{1}{6} \dddot{A}_{k} \eta^{3}+\ldots
$$

subject to the differential cquation

$$
\dot{A}=A \Omega ;
$$

\footnotetext{
${ }^{(2)}$ J. W. Jordan, An Accurate Strapdown Direction Cosine Algorithm, NASA TND-5384, 1969.
} 
then

$$
\begin{aligned}
& \ddot{A}=\dot{A} \Omega+A \dot{\Omega}=A\left(\Omega^{2}+\dot{\Omega}\right) \\
& \dddot{A}=A\left[\Omega^{3}+\Omega \dot{\Omega}+2 \Omega \dot{\Omega}+\ddot{\Omega}\right] .
\end{aligned}
$$

giving

$$
A_{k+1}=A_{k}\left[I+\Omega_{k} \eta+\frac{1}{2}\left(\Omega_{k}^{2}+\dot{\Omega}_{k}\right) \eta^{2}+\frac{1}{6}\left(\Omega_{k}^{3}+\dot{\Omega}_{k} \Omega_{k}+2 \Omega_{k} \dot{\Omega}_{k}+\dot{\Omega}_{k}\right) \eta^{3}+\ldots\right]
$$

However,

$$
\Omega_{\mathrm{k}} \eta+\frac{1}{2} \dot{\Omega}_{\mathrm{k}} \eta^{2}+\frac{1}{6} \ddot{\Omega}_{\mathrm{k}} \eta^{3}+\ldots
$$

is just the Taylor Series expansion of the gyro data $\delta$. Then,

$$
A_{k+1}=A_{k}\left[I+\delta+\frac{1}{2} \Omega_{k}^{2} \eta^{2}+\frac{1}{6}\left(\Omega_{k}^{3}+\dot{\delta}_{k} \Omega_{k}+2 \Omega_{k} \dot{\delta}_{k}\right) \eta^{3}+\ldots\right] .
$$

Since only the first derivatives of the $\Omega$ matrix appear in the third order expansion, it is clear that any computations based on this expansion will be limited by the piecewise linear $\underline{\omega}$ theory.

Third Order @uaternion Fixpansion

Precisely the same results occur as for the direction cosine expansion.

\section{Comparison}

Computations using each of the above methods give results closely approximating the theoretical limit for piecewise linear $\underline{\omega}$. The Jordan algorithm results in much less computer loading, however, and 18 therefore to be prefergert. 
DISTRIBUTION:

1321 C. E. Dalton

1322 R. D. Andreas

2136 C. R. Blaine

2300 J. C. King

2320 K. L. Gillespie

2326 G. M. Heck

2326 J. N. Shinkle (10)

8266 E. A. Aas

3141 C. A. Pepmueller (Actg) (5)

3151 W. L. Garner (3)

For ERDA/TIC (Unlimited Release)

ERDA/TIC (25)

(R. P. Campbell, 3172-3) 